\title{
Targeting the bacterial SOS response for new antimicrobial agents: drug targets, molecular mechanisms and inhibitors
}

\begin{abstract}
Antimicrobial resistance is a pressing threat to global health, with multidrug-resistant pathogens becoming increasingly prevalent. The bacterial SOS pathway functions in response to DNA damage that occurs during infection, initiating several pro-survival and resistance mechanisms such as DNArepair and hypermutation. This makes SOS pathway components potential targets that may combat drug-resistant pathogens and decrease resistance emergence. This review discusses the mechanism of the SOS pathway, the structure and function of potential targets AddAB, RecBCD, RecA and LexA, and efforts to develop selective small-molecule inhibitors of these proteins. These inhibitors may serve as valuable tools for target validation and provide the foundations for desperately needed novel antibacterial therapeutics.
\end{abstract}

\section{Key words}

Antimicrobial resistance; drug discovery; SOS response

\section{Introduction}

The emergence of multi-drug resistant pathogenic bacteria is one of the most serious threats to human health globally. Antimicrobial resistance (AMR) has the potential to render numerous lifesaving medical advances, such as surgery and chemotherapy, so dangerous as to be impractical [1]. The challenge of rising AMR levels is compounded by a lack of new classes of antibiotics in development. Indeed, many compounds recently developed or currently in development are modifications of known classes of antibiotics [2], and as such may be readily compromised be existing resistance mechanisms [3]. The Enterococcus faecium, Staphylococcus aureus, Klebsiella pneumoniae, Acinetobacter baumannii, Pseudomonas aeruginosa, and Enterobacter species (so called 'ESKAPE' pathogens) are the main cause of nosocomial infections globally, and commonly exhibit minimum inhibitory concentrations (MICs, see Defined Key Terms) above the breakpoint of multiple antibiotics [4]. The paucity of novel antibiotics in development is a result of both technical challenges for compound development and financial barriers, such as short treatment courses. New antibiotics must also be kept in reserve for treatment of resistant infections to prolong clinical utility. This results in an apparent dichotomy where critically needed new antibiotics have limited markets and therefore decreased financial incentives for development [3].

There is therefore an urgent need for antibiotics with novel mechanisms of action (MOAs) that function through new targets, and various strategies have been employed in recent years towards this end [5-8]. However, new molecules that inhibit bacterial viability may be expected to drive resistance through selection pressures in both clinical and environmental settings [9]. Alternatively, molecules that inhibit bacterial pathways that cause resistance may increase the lifetime of existing therapies and/or decrease the ability of pathogens to survive in infection settings. One such pathway is the bacterial 'SOS' pathway. The bacterial SOS response is activated by DNA damage by antibiotic treatment [10] or attack by host immune systems [11], and promotes both bacterial survival and the emergence of resistance via mutagenic DNA repair [12]. Resistance can arise through mutation of the genes encoding antibiotic targets that alter the binding site, horizontal gene transfer of resistance determinants, or non-genetic changes such as persister or biofilm formation [13]. SOS inhibition may therefore decrease resistance emergence against antibiotics in co-therapies, and increase the potency of DNA-damaging antibiotics where resistance exists. SOS inhibitors would also be expected to sensitise pathogens to host immune defences, such as the respiratory burst of neutrophils. The high 
conservation of pathway components in bacteria means that inhibitors may possess broad-spectrum activity in a variety of pathogens [14].

Interest in SOS inhibition is increasing [15-18], although it is yet to be determined what the optimum target(s) within this pathway are and the best antibiotic partner(s) for SOS-inhibiting adjuvants [19]. This review will therefore describe the mechanistic steps involved in activation of the SOS response along with efforts to develop inhibitors of key enzymes in the pathway. These small molecule inhibitors may serve as valuable tools to demonstrate the therapeutic benefit of SOS response inhibition or as intermediates toward potential antibiotic adjuvants, which are much needed in the global fight against AMR.

\section{The SOS pathway}

The SOS pathway is a widely conserved stress response in bacteria that is activated by DNA damage and plays a central role in survival and resistance evolution during stress [19]. The SOS response is also involved in several processes related to pathogenesis, including dissemination of virulence factors [20], production of small colony variants (SCVs) [12], and horizontal gene transfer [21]. The SOS pathway is activated by DNA damage caused by stalled DNA replication, antibiotic treatment including at sub-minimum inhibitory concentrations, and oxidative stress for example from attack by host immune responses $[12,22,23]$. Importantly, the action of the SOS response means that bacteria may develop resistance not only to the direct source of cellular stress; for example, expression of lowfidelity DNA polymerases increases mutation rates and can lead to acquisition of resistance to antibiotics beyond that which caused the DNA damage $[22,23]$. As such, the sos response is a powerful mechanism for the development of multidrug resistance.

One of the most commonly studied mechanisms for SOS induction is through production of DNA double stand-breaks (DSB), for example by the action of quinolone antibiotics which inhibit DNA gyrase and topoisomerase IV. A single DNA DSB can be lethal if not repaired during cell replication, and bacteria possess efficient methods of DSB repair (Figure 1). The DSB is loaded with AddAB or RecBCD helicase-nuclease enzymes for processing to produce a 3' ssDNA strand onto which RecA is loaded [24]. The RecA-ssDNA filament promotes invasion of intact homologous dsDNA to continue repair of the DSB [25]. If the interacting DNA strands differ genetically, then this DSB repair mechanism can generate genetic recombinants and drive evolution [26]. In addition to homologous recombination, RecA interacts with LexA repressor proteins causing cleavage of LexA and initiates expression of SOS-responsive genes (Figure 1). The number and type of genes regulated by LexA cleavage varies among bacterial species [22], with $>16$ genes regulated in $S$. aureus [27] compared to $>40$ genes in E. coli [28].

LexA-regulated proteins perform a diverse range of functions in response to DNA damage, such as DNA base excision, homologous recombination, translesion DNA replication, and arrest of cellular replication, along with a range of as-yet unidentified functions [29]. Interestingly, SOS responsive genes are induced in a temporal fashion depending on the duration of DNA stress [30]. Initially, genes involved in nucleotide excision repair and error-free replication are induced; subsequently, homologous recombination repair genes are expressed; finally, genes involved in error-prone polymerases are expressed along with the cell division inhibitor protein (SulA) to slow growth and allow extra time for mutation acquisition [13, 29]. LexA is under the control of an SOS-inducible promoter, therefore production of LexA during the SOS process ensures LexA repression will downregulate SOS gene expression upon removal of cellular DNA stress [31]. Bacterial populations may benefit from stochastic activation of DNA repair that leads to mutagenesis in a subpopulation of 
cells while maintaining the genome of most other cells [32]. Single-cell imaging of wild-type $E$. coli estimates mutation rates at 0.0022 mutation/hour in the absence of selection pressures [33].

Various genetic studies have shown therapeutically beneficial phenotypes result from disruption of the SOS pathway components. Loss of RecB or RecC function in E. coli increases susceptibility to the quinolone antibiotic ciprofloxacin (CFX), and recC knockout partially restores CFX sensitivity in E. coli possessing a DNA gyrase mutation (S83L) which confers $\mathrm{CFX}$ resistance [34]. AddAB is required for SOS induction in response to antibiotic (co-trimoxazole) and oxidative $\left(\mathrm{H}_{2} \mathrm{O}_{2}\right)$ stress in $\mathrm{S}$. aureus to initiate mutagenic DNA repair and increase resistance rates [10, 12]. RecA deletion in E. coli and S. aureus increases sensitivity to levofloxacin and reduces antibiotic-induced resistance [35]. A systematic analysis of an E. coli $\triangle r e c A$ mutant strain exposed to various chemical compounds indicated increased sensitivity to 28 compounds with diverse biological targets [36]. Loss of autocleavage function in $E$. coli LexA increases sensitivity to multiple antibiotics, such as quinolones, mitomycin $C$, and nitrofurantoin [19]., and enhances bacterial killing and reduces resistance following treatment with CFX or other antibiotics in murine infection models $[27,37]$. The SOS response also regulates virulence, for example in Vibrio cholerae SOS regulates CTX $\phi$ phage production that encodes for the cholera toxin and LexA cleavage is required for stimulation of CTX $\phi$ production [20].

The SOS pathway therefore offers multiple potential targets in $\operatorname{RecBCD}, A d d A B$, RecA and LexA that may be inhibited to potentiate killing by DNA-damaging antibiotics, host immune defences and potentially decrease the rate of resistance emergence. The biochemical mechanisms of these enzymes indicate there may be multiple potential sites for targeting with small molecules, and structural information is available for each enzyme that may help guide inhibitor development.
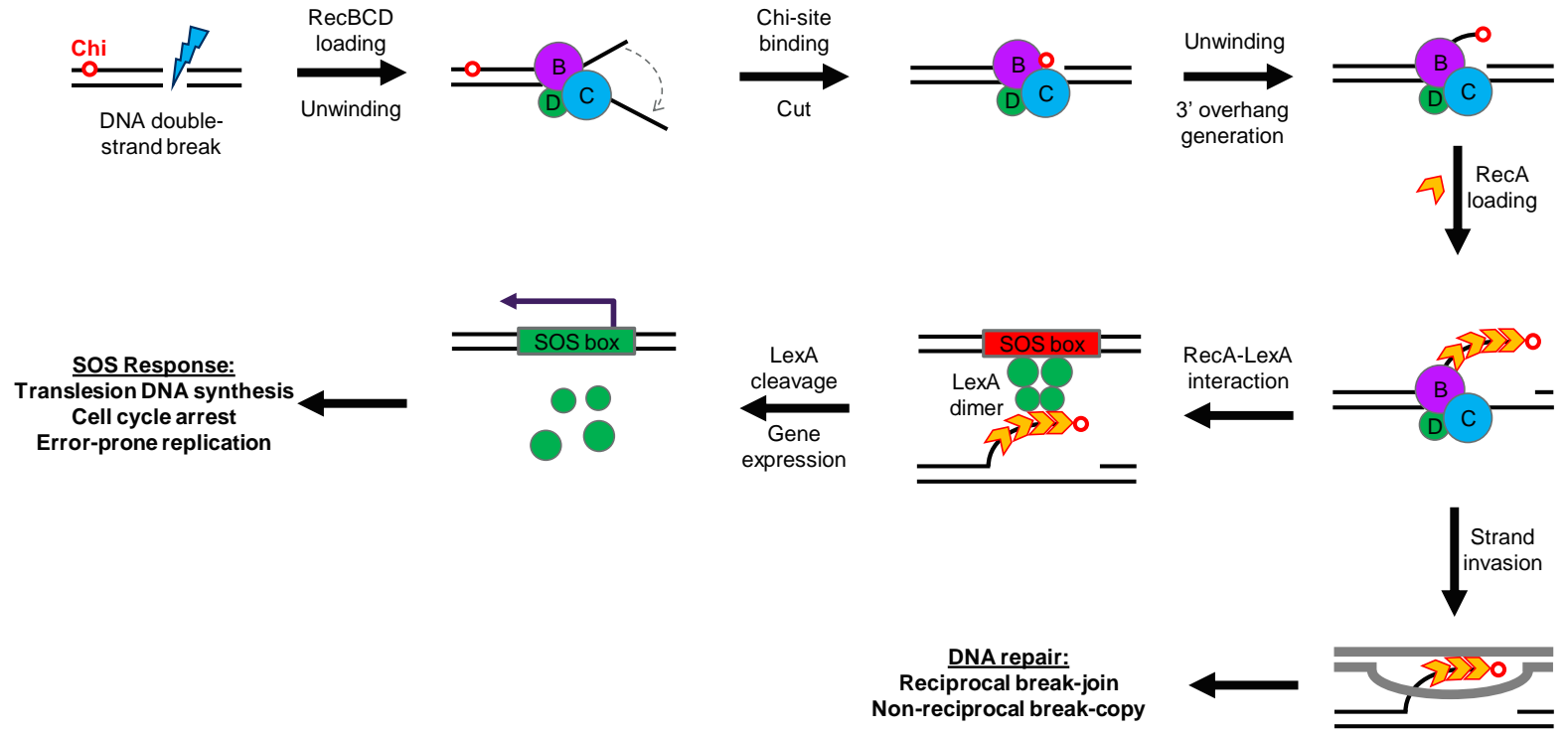

Figure 1. The bacterial DNA repair and SOS pathway. Cellular stresses such as DNA-damaging antibiotics induce DNA double-strand breaks (DSBs). The first step in DSB repair and DSB-stimulated recombination is RecBCD binding to a DNA end. The RecBCD helicase-nuclease complexes load onto the DSB and unwind and cut the DNA duplex to generate a 3' single-strand DNA (ssDNA) overhang. AddAB performs a similar function to RecBCD via a different mechanism as a result of differences in the helicase-nuclease subunits between the two enzymes. Multiple copies of RecA then bind to the ssDNA to form a RecA-nucleofilament (RecA*), which promotes the invasion of dsDNA (adapted from mechanism proposed in [38]). In addition, RecA* interaction with LexA repressor proteins triggers LexA autocleavage resulting in the activation of transcription and expression of SOSresponsive genes.

\section{RecBCD and AddAB mechanisms and inhibitors}


RecBCD is a trimeric complex of $\sim 330 \mathrm{kDa}$ containing both helicase and nuclease subunits. Structural studies have provided insights into the molecular mechanisms underlying RecBCD function, showing central channels through which dsDNA enters the complex and ssDNA is extruded (Figure 2A) [39-41]. RecBCD contains two ATP-dependent helicase subunits; RecD is a $5^{\prime}-3^{\prime}$ helicase, whereas RecB is a $3^{\prime}-$ 5 ' helicase and also contains a nuclease domain [24]. The RecBCD complex rapidly unwinds the DNA duplex at up to 1,500 base pairs (bp) per second, over sequences up to 100,000 bp in length [42]. RecB and RecD bind to the $3^{\prime}$ and $5^{\prime}$ strands of a DSB[43], respectively, pulling the two DNA strands across a 'pin' in RecC, splitting the duplex into single strands [40]. Electron microscopy shows inactivation of either helicase causes a DNA loop to be produced from the complex [44]. As the RecD subunit moves along the $5^{\prime}$ strand more rapidly than RecB along the $3^{\prime}$ strand, a ssDNA loop is produced on the $3^{\prime}$ strand, presumably ahead of the complex $[42,45]$. There is debate about the exact mechanism of RecBCD DNA processing prior to recognition of a crossover hotspot instigator (Chi) site $[24,46]$. The nuclease activity of RecB is dependent on $\mathrm{Mg}^{2+}$ and ATP concentrations; when $\mathrm{Mg}^{2+}$ concentrations are higher than ATP concentrations, RecBCD cuts both SSDNA strands as they exit the complex until a Chi site is reached, terminating $3^{\prime}$ digestion. When ATP concentrations are higher than $\mathrm{Mg}^{2+}$ concentrations, the RecBCD nuclease activity is modestly decreased and unwinding pauses at a Chi site to allow DNA cleavage of the $3^{\prime}$-ended strand (that with the Chi octamer 5' GCTGGTGG 3'), with continued unwinding producing the $3^{\prime}$ overhang. The mechanism used in cells will depend on the cellular $\mathrm{Mg}^{2+} /$ ATP concentrations. However, whilst the net effect of either mechanism is the production of 3' ssDNA for RecA loading, other consequences such as reciprocal recombination are not possible if there is degradation up to Chi or cutting of both strands at Chi (for more detailed discussions see $[24,46,47])$. Chi-site recognition occurs in the RecC tunnel $[48,49]$, which causes RecC to signal RecD to stop unwinding DNA and in turn, $\operatorname{Rec} D$ signals to $\operatorname{RecB}$ inducing a conformational change that swings the RecB nuclease domain into proximity of the $3^{\prime}$ strand $[50,51]$. This results in cutting a few nucleotides to the $3^{\prime}$ side of the Chi octamer [50,52]. The RecB nuclease loads RecA onto the 3' ssDNA overhang [53] and it has been proposed that RecB subsequently swings away from the DNA, preventing further cutting $[51,54]$. This enables homologous recombination and SOS activation by the RecA-DNA nucleofilament (RecA*).

$A d d A B$ is a dimeric complex of $\sim 270 \mathrm{kDa}$, which performs an analogous function to RecBCD in DSB processing, although the mechanism of processing differs from RecBCD. Structural studies of AddAB have also provided insights into the mechanism of this complex, indicating both similarities and differences with $\operatorname{RecBCD}[55,56]$. AddAB unwinds DNA at rates of up to $1,600 \mathrm{bp} / \mathrm{s}$ with a processivity in excess of 30,000 bp [57]. The AddA subunit possesses a similar structure to RecB, containing a 3'-5' helicase and also nuclease domain, and the $A d d B$ subunit performs an analogous chi-scanning function to $\operatorname{RecC}[48,55]$. AddB also contains a nuclease domain, but there is no homologue of RecD performing 5 '- 3 ' helicase function. AddAB therefore contains two nuclease and one helicase subunits, compared to the two helicase and one nuclease subunits of RecBCD [24]. Recent discovery of the related AdnAB complex in mycobacterial species which contains two helicase and two nuclease subunits suggests a common evolutionary origin for $A d d A B$ and RecBCD [24]. AddAB is required for recombinational DSB repair, but it is unknown if the complex has an active role in loading RecA, similar to RecBCD [14].

Either $A d d A B$ or RecBCD is present in most sequenced bacteria, but these complexes are not found in humans [14], making these attractive targets for inhibitor development. Inhibitors of RecBCD exist in nature, for example in bacteriophages where the action of RecBCD would otherwise digest phage DNA. The phage $\lambda$ protein Gam inhibits RecBCD, and structural studies show that the protein contains a negatively charged $\alpha$-helical subunit that inserts into the RecBCD channel that can be occupied by the double-stranded DNA substrate [58]. The tight Gam-RecBCD interaction over $2500 \AA^{2}$ sterically blocks access of DNA to the active sites, and correspondingly expression of Gam proteins in various 
bacterial strains increases susceptibility to DNA-damaging quinolone antibiotics [58]. The presence of multiple functional sites in the $A d d A B$ and RecBCD complexes provides several sites for targeting with small-molecule inhibitors, including nuclease, helicase, and ATPase sites, along with other more challenging sites such as protein-protein or protein-DNA interaction interfaces. This has resulted in several attempts to identify inhibitors of these complexes as potential antibiotic agents.

Initial $A d d A B / R e c B C D$ inhibitor discovery efforts identified molecules with non-selective MOAs. Adozelesin, Ecteinascidin 743 and Hedamycin were reported to inhibit the helicase, nuclease and ATPase activities of RecBCD by covalently reacting with DNA to cause DNA structural distortions [59]. However, such mechanisms of action make these compounds unsuitable for therapeutic applications owing to potential off-target effects and high cytotoxicity as a result of DNA alkylation [60]. Similarly, cisplatin and psoralen inhibit RecB [61] and RecBCD [62], respectively, due to their DNA alkylation abilities, but are also unsuitable starting points for development of specific AddAB/RecBCD inhibitors. Non-specific inhibitors provide little utility as tool molecules for use in target validation studies, which are a key step prior to focussed drug discovery efforts. There have, however, been efforts in recent years to identify small-molecules with more specific MOAs.

A screen for inhibitors of RecBCD helicase-nuclease activity by Achaogen identified the small-molecule NSAC1003 (Figure 2B). RecBCD nuclease assays with varied ATP concentrations, along with in silico docking, suggest that NSAC1003 competes for the ATP pocket of RecBCD, thus inhibiting ATPdependent helicase and therefore also nuclease activity $\left(\mathrm{IC}_{50} \sim 6 \mu \mathrm{M}\right)$ [26]. Intriguingly, NSAC1003 appears to slow the RecB helicase motor compared to RecD, behaviour also observed in two RecB mutations in the ATP-binding site (Y803H and V804E) [38]. This results in RecB nuclease cuts at nonChi site positions that vary in response to NSAC1003 concentration [26]. There remains scope for optimisation of the properties of NSAC1003, alongside investigation of the inhibitory MOA which may provide further insights into RecBCD enzymology. The selectivity of an ATP-competitive RecBCD inhibitor within a cellular context will be a consideration in future development.

Further efforts at identification of small-molecule inhibitors of $A d d A B / R e c B C D$ have resulted in discovery of five alternative chemical series [63]. A cellular assay using $E$. coli infected with a phage T4 gene 2 deficient mutant, lacking the phage's RecBCD inhibitor protein, was used to identify inhibitors of $E$. coli RecBCD, or Helicobacter pylori AddAB expressed in a recB21 null mutant background. In this assay inhibition of AddAB or RecBCD activity prevents digestion of the phage DNA and results in the phage killing the host cell. A high-throughput screen (HTS) screen of $>325,000$ compounds was conducted, with counter-screening for direct effects on cell growth alongside assaying for inhibition of purified AddAB and RecBCD exonuclease activity [63]. This resulted in identification of five distinct chemotypes of potential inhibitors, which underwent further SAR exploration. One of the most potent compounds, CID1045135 (Figure 2B), inhibits the helicase, nuclease, and Chi-cutting activities of RecBCD (nuclease $\mathrm{IC}_{50} \sim 13 \mu \mathrm{M}$ and Chi-cutting $\mathrm{IC}_{50} \sim 10 \mu \mathrm{M}$ ) and the nuclease activity of AddAB $\left(\mathrm{IC}_{50} \sim 34 \mu \mathrm{M}\right)$, and is $\sim 10$-fold more potent in intracellular assays [63]. The pipemidic acid (PA) moiety of CID1045135 suggests a potential binding mechanism that may function, in part, through intercalation of the DNA double helix. Indeed, PA is a known quinolone inhibitor of DNA gyrase and topoisomerase IV, where the inhibitor binds the enzyme-DNA complex through intercalation [64].

Scaffold hopping from PA to the improved fluoroquinolone intercalator CFX, along with rational SAR optimisation, generated IMP-1700 [16]. Compared to CID1045135, potentiation of CFX killing of methicillin-resistant S. aureus (MRSA) by IMP-1700 is increased $>160$-fold (cellular EC $50 \sim 6 \mathrm{nM}$ ). Despite the presence of a fluoroquinolone moiety, IMP-1700 does not inhibit DNA gyrase or topoisomerase IV, known quinolone targets. Further, IMP-1700 inhibited the SOS response in cellular reporter assays, and compound-functionalised Sepharose could successfully pull-down the 
recombinant $A d d A B$ complex. Collectively, these data support the highly potent potentiation of DNA damage in MRSA by IMP-1700 as occurring through inhibition of AddAB [16]. However, the cellular target(s) and binding mechanism of IMP-1700 remain to be robustly determined, therefore despite generating a highly promising phenotypic response at remarkably low concentrations, the MOA requires further investigation.

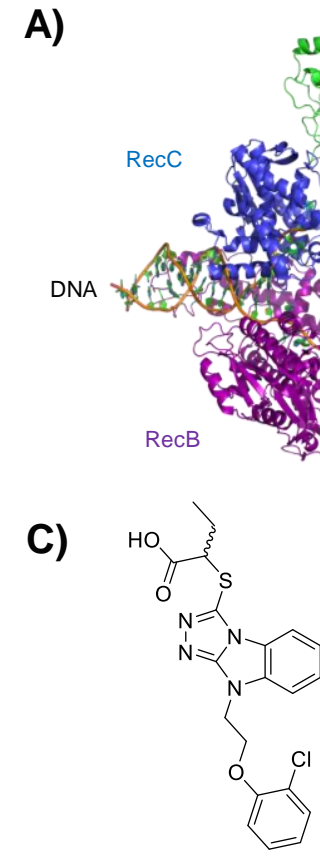

NSAC1003

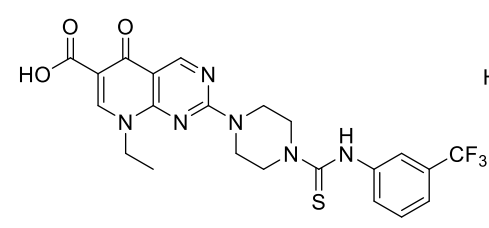

CID1045135
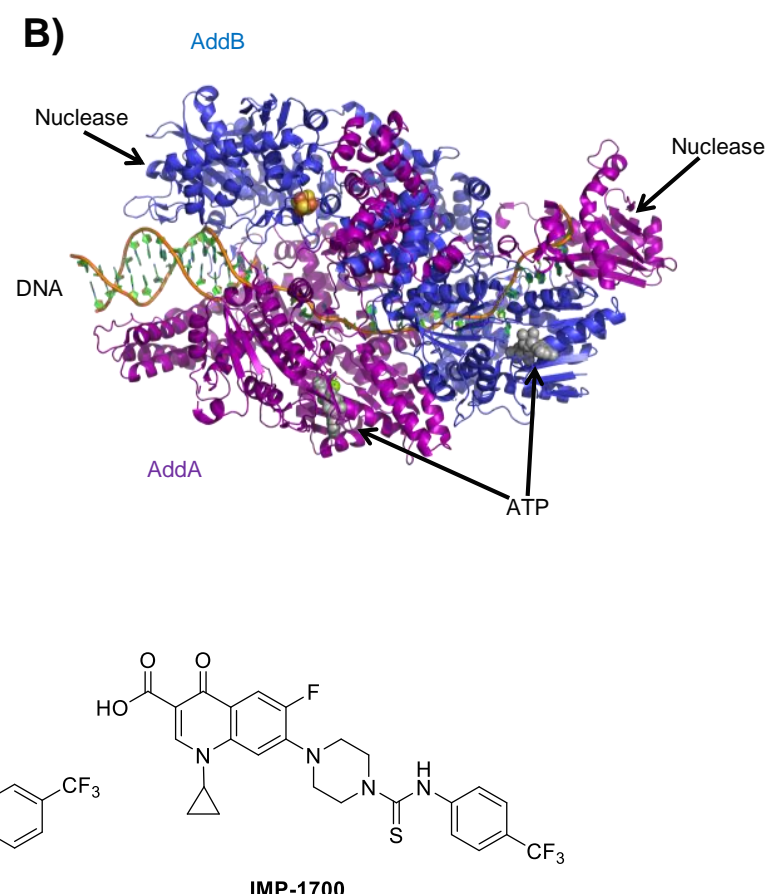

Figure 2. AddAB and RecBCD structures and small molecule inhibitors. A) Structure of RecBCD in complex with DNA showing nuclease subunit (PDB 1W36) [40]. B) Structure of AddAB in complex with DNA and nonhydrolysable ATP analogue ADPNP showing nuclease subunits and ATP binding sites (PDB 4CEJ) [55]. C) Structures of small-molecule AddAB/RecBCD inhibitors NSAC1003 [26], CID1045135 [63], and IMP-1700 [16].

\section{RecA mechanism and inhibitors}

RecA is essential for homologous recombination, and displays remarkable conservation in many organisms $[65,66]$. RecA is a $\sim 38 \mathrm{kDa}$ monomeric protein which mediates DNA strand exchange during homologous recombination, acts when complexed with ssDNA as a co-protease to catalyse cleavage of LexA $[67,68]$ and certain phage repressors [31], and forms an active mutasomal complex with DNA Polymerase $\mathrm{V}$ for translesion DNA synthesis [69]. Live bacterial cell single-molecule imaging shows RecA is predominantly located in storage bodies in the absence of cellular stress. Upon DNA damage, the storage bodies release RecA which oligomerises on ssDNA to form the RecA* nucleoprotein filament and SOS-signalling complexes. The RecA storage bodies reform after completion of DNA repair [70]. RecA contains two DNA binding sites and an ATPase site in the central domain of the protein, and X-ray crystal structures of RecA complexes with either ssDNA or a DNA heteroduplex have provided insights in the mechanism of homologous recombination [71] (Figure 3A). ATP and ssDNA cooperatively bind to the RecA-RecA interface, explaining the ATP-dependency of DNA binding and mechanism for release by ATP hydrolysis. For strand exchange in homologous recombination, dsDNA binds to a second RecA DNA-binding site causing stretching to disrupt base pairing, whilst the flexible ssDNA is bound in conformation resembling duplex DNA [71]. This allows sampling of one of the donor strands by base pairing with the SSDNA substrate. Alterations in the cellular nucleotide pool of $E$. coli containing a constitutively active RecA mutant affects RecA activity and the SOS response [72]. RecA is also implicated in swarming motility, a key factor in colonisation and pathogenesis, although this 
occurs through a mechanism independent of extensive canonical RecA* formation [73]. As with RecBCD, several protein inhibitors of RecA function are found in nature, such as bacterial RecX and Dinl, which can disrupt the function of RecA* and inhibit SOS induction [74], or RdgC which competes with RecA for DNA binding sites [75]. The ubiquity of RecA has led to the proposal that RecA may be a widely-applicable drug target for pathogenic bacteria [30], although selectivity over the human structural and functional analogue, Rad51, must be ensured.

The role of RecA in bacterial survival and resistance mechanisms has therefore led to numerous attempts to identify inhibitors, with varying degrees of success. Initial attempts to identify RecA inhibitors focussed on the use of nucleotide analogues aiming to exploit the shallow nature of the RecA ATPase pocket to achieve cellular selectivity $[76,77]$. Whilst nucleotide analogues could inhibit RecA in biochemical assays and decrease SOS induction in permeabilised cells, the charged pyrophosphate moiety limits cellular penetration and thus therapeutic utility [76, 77]. $\mathrm{Zn}^{2+}, \mathrm{Cu}^{2+}$ and $\mathrm{Hg}^{2+}$ induce aggregation of RecA in vitro [78] and $\mathrm{Zn}^{2+}$ inhibits the SOS response in E. coli, although this is at a concentration at which toxicity is observed in human skin cells [79], thus limiting therapeutic use in a practical sense. Solving the structure of RecA has also led to virtual screening and ligand development efforts [80, 81]; however, specific target inhibition in biochemical assays, structural validation and structure-activity relationships (SAR) consistent with predictions remain to be demonstrated for these proposed ligands.

The ATPase and DNA-filament assembly activity of RecA have been used to develop robust HTScompatible assay formats for screening of large panels of compounds [82]. However, small-molecule screens targeting ATPase activity identified hits such as curcumin, Congo Red, Suramin [83] or structurally related molecules [84]. Compounds such as these, termed pan-assay interference compounds (PAINS) [85], act through promiscuous or non-selective MOAs that prohibit therapeutic development. Other proposed RecA inhibitors that may non-specifically suppress the SOS response include Baicalein, a radical scavenger that decreases ROS in cells [86], and hits from RecA HTS efforts that contain PAINS motifs such as nitro aromatics and Michael acceptors [87]. It is therefore critical that new inhibitors be assessed for PAINS activity following modern guidelines [88, 89] in order to ensure investigation and use of molecules that have genuine therapeutic potential and which function in the proposed manner.

Other more promising RecA inhibitor chemotypes have been identified, for example BRITE-338733 (IC $\left.\mathrm{C}_{50} 4.7 \mu \mathrm{M}\right)$ [90] (Figure 3B), although compounds from this series typically exhibit steep Hill slopes $(>5)$ in inhibition dose-response curves. Steep Hill slopes are not definitive evidence of PAINS behaviour, but these can be a warning sign for non-specific MOAs [91]. Iron(III) phthalocyanine tetrasulfonic acid (Fe-PcTs) has recently been reported as an inhibitor of RecA, which potentiates bactericidal antibiotics and reduces resistance acquisition in in vivo infection models [17] (Figure 3B). However, further investigation is required to understand the target-binding and SAR of this series.

The N-terminal region of RecA has been used to design peptide-based inhibitors of RecA-RecA multimerization, with the peptide 'INPEP' (AcHN-YGGDKQKALAKALEKIAKQFGKVTVMRTT-CONH ${ }_{2}$ ) successfully inhibiting RecA ATPase activity $\left(\mathrm{IC}_{50} 35 \mu \mathrm{M}\right)$. Replacement of the core methionine residue with a cysteine residue disulphide bonded to a 2-thiopyridine moiety increased potency (INPEP-STP, $\mathrm{IC}_{50} 3 \mu \mathrm{M}$ ) by disulphide exchange of this covalent warhead with a cysteine in RecA (C116) [92]. The stability and selectivity of the disulphide warhead in a cellular context will be an important consideration for further development. An alternative peptide inhibitor based of an $\alpha$-helical motif in the RecX protein inhibitor of RecA has been developed, termed 4E1 [93]. The sequence $\left(\mathrm{H}_{2} \mathrm{~N}-\right.$ EEEEKVKILRYLLYRLIY- $\mathrm{CO}_{2} \mathrm{H}$ ) contains key residues from a RecX region which binds closely to the DNA substrate in the RecX-RecA-ssDNA complex, and thus 4E1-DNA interactions cannot be excluded from 
its MOA. 4E1 inhibits RecA ATPase and strand exchange in vitro, and also decreases UV resistance and SOS activation when expressed in E. coli [93]. Cell penetration and metabolic stability can present issues for development of peptide-based inhibitors; however, modern approaches in peptide cyclisation via chemical crosslinking ('stapling'), $\alpha$-helix mimetics or cell-penetrating sequences may address some of these limitations [94-96].

A)

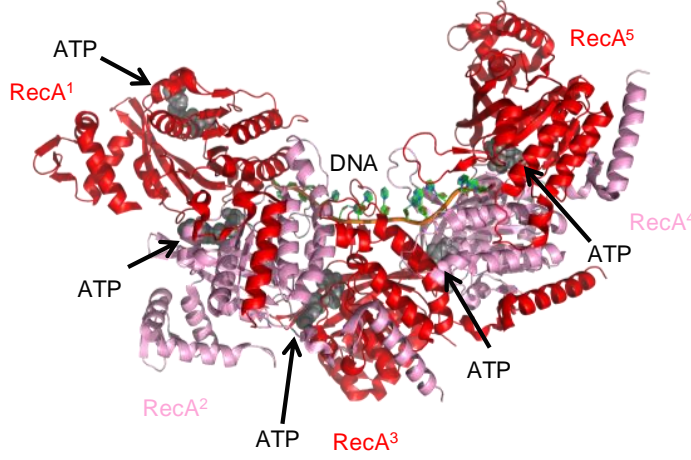

B)

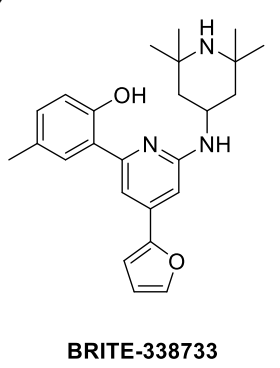

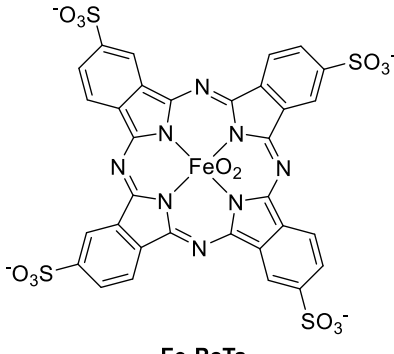

Fe-PcTs

Figure 3. RecA structure and small molecule inhibitors. A) Structure of five RecA monomers in complex with ssDNA (PDB 3CMW) showing binding sites for non-hydrolysable ATP analogue ADP-aluminium fluoride-Mg (ADP$\mathrm{AlF}_{4}-\mathrm{Mg}$ ) [71]. B) Inhibitors of RecA BRITE-338733 [90] and Iron(III) phthalocyanine tetrasulfonic acid (Fe-PcTs) [17].

\section{LexA mechanism and inhibitors}

Under normal conditions, LexA represses the transcription of many DNA repair proteins by binding to SOS 'boxes' in their operators. LexA binds as a dimer to the SOS box, a 20 bp palindromic DNA sequence of consensus sequence $\mathrm{CTGTN}_{8}$ ACAG [97], with high affinity (consensus linker (AT) 4 apparent $K_{\mathrm{d}} 0.8 \mathrm{nM}$ [98]). LexA consists of an N-terminal DNA-binding domain and C-terminal latent protease domain that is also responsible for dimerization. The LexA $A_{2}$-DNA co-crystal structure reveals that the DNA-binding domains interact with DNA in a canonical fashion through a winged helix-turnhelix (wHTH) motif [98] (Figure 4A). The recognition helix binds in the major groove of the DNA contacting the conserved residues of the SOS box. The wings contact the phosphodiester backbone on the adjacent minor groove, but unlike many dimeric WHTH proteins, point inwards to the central minor groove. The wings also form hydrophobic contacts with the DNA-binding domain of the other LexA subunit in the dimer [98]. The LexA DNA-binding and protease domains are connected by a flexible five amino acid linker [98, 99]. The peptide site of autocleavage (shown in red, Figure 4A) is positioned near the C-terminal protease active site (shown in grey, Figure 4A). DNA binding does not alter the position of the cleavage peptide, but interaction with RecA* filaments may stabilize a conformation where the cleavage-site is bound across the active site $[98,99]$. Autocleavage results in separation of the DNA-binding and protease domains, exposing recognition sequences for the ClpXP protease flanking the autocleavage site that are otherwise hidden in non-cleaved LexA [100]. While the different symmetry between LexA dimers and head-to-tail RecA filaments mean two docking events may be needed to cleave both LexA subunits, loss of one subunit may decrease DNA affinity sufficiently to disrupt repression [98]. This mechanism provides different sites for inhibition, including the protease catalytic site or RecA*-LexA interactions.

LexA is an attractive target for SOS inhibition as mammals do not possess LexA homologues. First-inclass inhibitors of LexA autoproteolysis were identified by a collaboration between the University of Pennsylvania and GlaxoSmithKline [15]. A fluorescence polarisation assay measuring LexA autoproteolysis activated by RecA* was used to screen 1.8 million compounds, with counter-screening performed using orthogonal biochemical assays and a cellular SOS reporter (GFP under control of a 
LexA promoter). Hits were curated to exclude PAINS activity, with mechanistic analysis suggesting that compound D1 likely inhibits LexA autoproteolysis, whereas compounds $\mathrm{C} 1$ and S5 likely interfere with the RecA*-LexA interaction [15] (Figure 4B). The quality of hit matter obtained highlights the importance of well-curated libraries that are free from PAINS, as well as the larger library sizes available in industrial settings [88]. Initial SAR exploration resulted in Compound 14, showing a modest increase in biochemical potency (LexA cleavage $\mathrm{IC}_{50} 9 \mu \mathrm{M}$ ), alongside disruption of cellular SOS response ( $\left.E_{50} 32 \mu \mathrm{M}\right)$, and encouragingly demonstrated decreased resistance emergence [18] (Figure 4B). The nucleophilic serine residue (S119) in the LexA active site may also allow for targeting with covalent ligands. (3-Aminophenyl)boronic acid has recently been identified as a covalent ligand of this active site residue [101], although further fragment growth is required. Protease active site inhibitors will have to overcome a high local substrate concentration by virtue of the cleavage loop being internal, and a large excess of nonspecific protease inhibitors, such as diisopropyl fluorophosphates, is required to inhibit LexA autoproteolysis [102].

A)

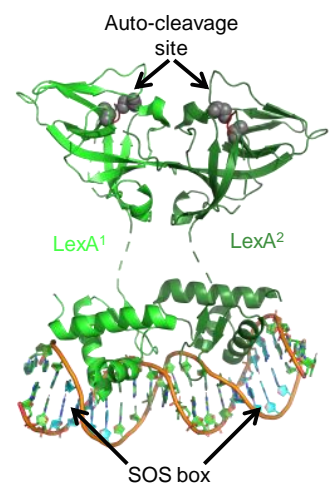

B)

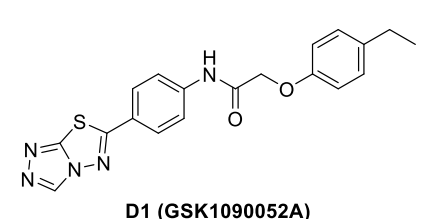

D1 (GSK1090052A)

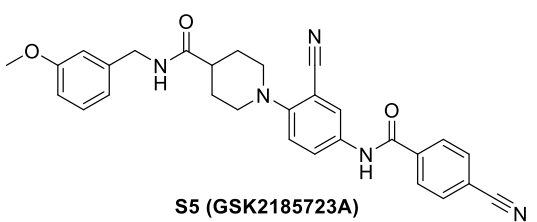

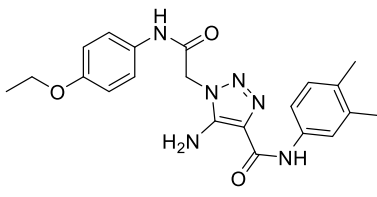

C1 (GSK1010702A)

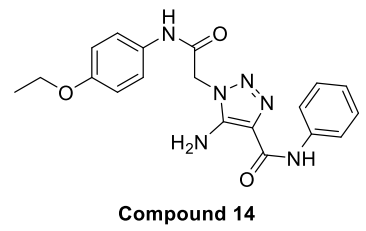

Figure 4. LexA structure and small molecule inhibitors. A) Structure of LexA dimer in complex with dsDNA containing an SOS-box sequence (blue), protease active site positions (grey) and site of autocleavage (red). The five residue linker (dashed line) between the DNA-binding and autocatalytic domains is too disordered to be modelled (PDB 3JSO) [98]. B) Small-molecule inhibitors of LexA, C1, D5, S5 [15] and Compound 14 [18].

\section{Executive Summary}

- $\quad A M R$ is an urgent threat that requires identification of new compounds functioning through novel MOAs to overcome technical barriers to drug development. Both academic and industrial research activity will be required to provide new validated targets and drive effective therapeutic development.

- Inhibitors of the bacterial SOS pathway may provide multiple potential beneficial uses, including potentiating DNA-damaging antibiotics, promoting immune clearance and blocking activation of resistance and virulence mechanisms.

- Inhibitors of key SOS pathway components AddAB/RecBCD, RecA, and LexA have been identified by biochemical and cellular screens. PAINS and non-specific MOAs represent pitfalls in any inhibitor discovery campaign, therefore confirmation of target engagement, quantitative drug synergy in co-therapies, and MOA validation in cells are critical to ensure translation of effective inhibitors.

- Existing SOS inhibitors have shown $\mathrm{nM}$ potency for potentiation of DNA-damaging antibiotics in cells (IMP-1700), decreased resistance emergence (Compound 14), and shown promising results in in vivo infection models (Fe-PcTs). These represent important tool molecules in investigating the validity of SOS pathway components as therapeutic targets.

- Despite solving of crystal structures for several key SOS pathway components, structural information for inhibitor-target complexes is lacking. This information can validate observed 
SAR trends and greatly accelerate inhibitor development through structure-guided approaches.

- Industry-academia collaboration has empowered successful identification of SOS inhibitors and continued engagement will be key to future progress.

\section{Future perspective}

The identification of inhibitors of SOS pathway components that show high potency and beneficial effects in a range of cellular and in vivo studies is an encouraging development in the global fight against AMR. However, key questions remain around robustly validating the MOA of these compounds in cells to build confidence in the tractability of this approach. Structural information remains a highly sought-after goal for SOS inhibitors that will expedite future compound development, and this may be achieved in coming years through structural biology or mass spectrometry-based binding-site identification. Further, important milestones remain to be met in compound stability, toxicity and pharmacokinetics of any new SOS inhibitors. The development of clinically-applicable SOS inhibitors may open new avenues for treatment of infections, however this also raises important questions. SOS inhibitors may sensitise bacteria resistant to DNA-damaging antibiotics, but this will not be applicable to all classes of antibiotics. Although suppression or delay of resistance emergence by SOS inhibition may be broadly applicable, understanding the drivers of resistance (e.g. mutation, horizontal gene transfer, persister formation) in a given infection will be a key determinant for potential use in the clinic. Identification of valid co-administration strategies therefore remains an important goal for future research. The financial challenges to antibiotic development mean these efforts may initially require investment from government and non-profit organisations to drive progress. The potential wider market for SOS inhibitors as therapeutic adjuvants that could be used with multiple classes of existing antibiotics and against several different pathogens may make this approach commercially attractive. The identification of technically tractable and financially viable SOS inhibitors may therefore be an important route to address the global challenge of AMR.

\section{Defined key terms}

\section{SOS response}

The bacterial response to DNA damage resulting in activation of a range of pathways that drive cellular survival and/or mutation to overcome cellular stress.

\section{Minimum inhibitory concentration (MIC)}

The lowest concentration of an antibiotic that prevents visible growth of an organism on agar or in broth cultured in the lab, used as a measure of the susceptibility of a given bacterium to an antibiotic.

\section{Breakpoint}

The highest plasma concentration of a given drug that can safely be achieved in a patient, which therefore defines whether a defined pathogen will survive or be resistant to an antibiotic treatment.

\section{Small colony variant}

Bacteria forming colonies approximately $10 \%$ of the size of wild-type strains, which have complex growth requirements from a variety of metabolic alterations resulting in slow growth. The basis of this phenotype is not fully understood, but is associated with intracellular persistence and reduced antimicrobial susceptibility. 
Persisters are non-hereditary subpopulations of antibiotic-tolerant cells that are not killed during treatment, and often show decreased or arrested cellular growth. Growth resumes when the cellular stress is removed. Persisters are not preexisting dormant cells, but rather their presence is induced by the SOS response.

\section{Pan-assay interference compounds (PAINS)}

Small-molecules that often give false-positive results for inhibition in a range of assays as a result of non-specific interactions with target biomolecules or interference with assay readouts. Development and/or investigation of PAINS molecules can result in substantial wasted research efforts, and such activity should therefore be vigorously controlled for.

\section{References}

1. Chandler CIR. Current accounts of antimicrobial resistance: stabilisation, individualisation and antibiotics as infrastructure. Palgr Commun 5 (2019).

2. Fischbach MA, Walsh CT. Antibiotics for emerging pathogens. Science 325(5944), 1089-1093 (2009).

3. Livermore DM, British Society for Antimicrobial Chemotherapy Working Party on the Urgent Need: Regenerating Antibacterial Drug D, Development. Discovery research: the scientific challenge of finding new antibiotics. J. Antimicrob. Chemother. 66(9), 1941-1944 (2011).

4. Santajit S, Indrawattana N. Mechanisms of Antimicrobial Resistance in ESKAPE Pathogens. Biomed Res Int 20162475067 (2016).

5. Evans LE, Krishna A, Ma Y et al. Exploitation of Antibiotic Resistance as a Novel Drug Target: Development of a beta-Lactamase-Activated Antibacterial Prodrug. J. Med. Chem. 62(9), 4411-4425 (2019).

6. Gray DA, Wenzel M. Multitarget Approaches against Multiresistant Superbugs. ACS Infect Dis 6(6), 1346-1365 (2020).

7. Imai Y, Meyer KJ, linishi A et al. A new antibiotic selectively kills Gram-negative pathogens. Nature 576(7787), 459-464 (2019).

8. Ling LL, Schneider T, Peoples AJ et al. A new antibiotic kills pathogens without detectable resistance. Nature 517(7535), 455-459 (2015).

9. Kinch MS, Patridge E, Plummer M, Hoyer D. An analysis of FDA-approved drugs for infectious disease: antibacterial agents. Drug Discov. Today 19(9), 1283-1287 (2014).

10. Clarke RS, Bruderer MS, Ha KP, Edwards AM. RexAB is essential for the mutagenic repair of Staphylococcus aureus DNA damage caused by co-trimoxazole. Antimicrob Agents Chemother doi:10.1128/AAC.00944-19 (2019).

11. Ha KP, Clarke RS, Kim GL et al. Staphylococcal DNA Repair Is Required for Infection. mBio 11(6), (2020).

12. Painter KL, Strange E, Parkhill J, Bamford KB, Armstrong-James D, Edwards AM. Staphylococcus aureus adapts to oxidative stress by producing $\mathrm{H} 2 \mathrm{O} 2$-resistant small-colony variants via the SOS response. Infect. Immun. 83(5), 1830-1844 (2015).

13. Culyba MJ, Mo CY, Kohli RM. Targets for Combating the Evolution of Acquired Antibiotic Resistance. Biochemistry 54(23), 3573-3582 (2015).

14. Cromie GA. Phylogenetic ubiquity and shuffling of the bacterial RecBCD and AddAB recombination complexes. J. Bacteriol. 191(16), 5076-5084 (2009).

15. Mo CY, Culyba MJ, Selwood T et al. Inhibitors of LexA Autoproteolysis and the Bacterial SOS Response Discovered by an Academic-Industry Partnership. ACS Infect Dis 4(3), 349-359 (2018). ** Reports the identification and validation of first in class small-molecule inhibitors of LexA autoproteolysis. 
16. Lim CSQ, Ha KP, Clarke RS et al. Identification of a potent small-molecule inhibitor of bacterial DNA repair that potentiates quinolone antibiotic activity in methicillin-resistant Staphylococcus aureus. Bioorg. Med. Chem. 27(20), 114962 (2019). ** Reports the development of IMP-1700, a highly potent potentiator of DNA damage and inhibitor of the SOS response in $S$. aureus.

17. Alam MK, Alhhazmi A, Decoteau JF, Luo Y, Geyer CR. RecA Inhibitors Potentiate Antibiotic Activity and Block Evolution of Antibiotic Resistance. Cell Chem Biol 23(3), 381-391 (2016).

18. Selwood T, Larsen BJ, Mo CY et al. Advancement of the 5-Amino-1-(Carbamoylmethyl)-1H1,2,3-Triazole-4-Carboxamide Scaffold to Disarm the Bacterial SOS Response. Front Microbiol 92961 (2018).

19. Mo CY, Manning SA, Roggiani M et al. Systematically Altering Bacterial SOS Activity under Stress Reveals Therapeutic Strategies for Potentiating Antibiotics. mSphere 1(4), (2016).

20. Quinones $\mathrm{M}$, Kimsey HH, Waldor MK. LexA cleavage is required for CTX prophage induction. Mol. Cell 17(2), 291-300 (2005).

21. Beaber JW, Hochhut B, Waldor MK. SOS response promotes horizontal dissemination of antibiotic resistance genes. Nature 427(6969), 72-74 (2004).

22. Baharoglu Z, Mazel D. SOS, the formidable strategy of bacteria against aggressions. FEMS Microbiol Rev 38(6), 1126-1145 (2014).

23. Tattevin P, Basuino L, Chambers HF. Subinhibitory fluoroquinolone exposure selects for reduced beta-lactam susceptibility in methicillin-resistant Staphylococcus aureus and alterations in the SOS-mediated response. Res. Microbiol. 160(3), 187-192 (2009).

24. Wigley DB. Bacterial DNA repair: recent insights into the mechanism of RecBCD, AddAB and AdnAB. Nat. Rev. Microbiol. 11(1), 9-13 (2013).

25. Dixon DA, Kowalczykowski SC. Homologous pairing in vitro stimulated by the recombination hotspot, Chi. Cell 66(2), 361-371 (1991).

26. Karabulut AC, Cirz RT, Taylor AF, Smith GR. Small-molecule sensitization of RecBCD helicasenuclease to a Chi hotspot-activated state. Nucleic Acids Res. 48(14), 7973-7980 (2020).

27. Cirz RT, Jones MB, Gingles NA et al. Complete and SOS-mediated response of Staphylococcus aureus to the antibiotic ciprofloxacin. J. Bacteriol. 189(2), 531-539 (2007).

28. Courcelle J, Khodursky A, Peter B, Brown PO, Hanawalt PC. Comparative gene expression profiles following UV exposure in wild-type and SOS-deficient Escherichia coli. Genetics 158(1), 41-64 (2001).

29. Maslowska KH, Makiela-Dzbenska K, Fijalkowska IJ. The SOS system: A complex and tightly regulated response to DNA damage. Environ Mol Mutagen 60(4), 368-384 (2019).

30. Pavlopoulou A. RecA: a universal drug target in pathogenic bacteria. Front Biosci (Landmark Ed) 23 36-42 (2018).

31. Walker GC. Mutagenesis and inducible responses to deoxyribonucleic acid damage in Escherichia coli. Microbiol Rev 48(1), 60-93 (1984).

32. Vincent MS, Uphoff S. Bacterial phenotypic heterogeneity in DNA repair and mutagenesis. Biochem. Soc. Trans. 48(2), 451-462 (2020).

33. Robert L, Ollion J, Robert J, Song X, Matic I, Elez M. Mutation dynamics and fitness effects followed in single cells. Science 359(6381), 1283-1286 (2018).

34. Tamae C, Liu A, Kim K et al. Determination of antibiotic hypersensitivity among 4,000 singlegene-knockout mutants of Escherichia coli. J. Bacteriol. 190(17), 5981-5988 (2008).

35. Singh R, Ledesma KR, Chang KT, Tam VH. Impact of recA on levofloxacin exposure-related resistance development. Antimicrob Agents Chemother 54(10), 4262-4268 (2010).

36. Maeda T, Horinouchi T, Sakata N, Sakai A, Furusawa C. High-throughput identification of the sensitivities of an Escherichia coli DeltarecA mutant strain to various chemical compounds. $J$ Antibiot (Tokyo) 72(7), 566-573 (2019).

37. LuTK, Collins JJ. Engineered bacteriophage targeting gene networks as adjuvants for antibiotic therapy. Proc Natl Acad Sci U S A 106(12), 4629-4634 (2009). 
38. Amundsen SK, Taylor AF, Reddy M, Smith GR. Intersubunit signaling in RecBCD enzyme, a complex protein machine regulated by Chi hot spots. Genes Dev. 21(24), 3296-3307 (2007).

39. Saikrishnan K, Griffiths SP, Cook N, Court R, Wigley DB. DNA binding to RecD: role of the 1B domain in SF1B helicase activity. EMBO J. 27(16), 2222-2229 (2008).

40. Singleton MR, Dillingham MS, Gaudier M, Kowalczykowski SC, Wigley DB. Crystal structure of RecBCD enzyme reveals a machine for processing DNA breaks. Nature 432(7014), 187-193 (2004). * Reports the crystal structure of RecBCD bound to a blunt-ended DNA hairpin, providing novel insights into the helicase, nuclease and Chi-recognition mechanisms of the complex.

41. Wilkinson $\mathrm{M}$, Chaban $\mathrm{Y}$, Wigley DB. Mechanism for nuclease regulation in RecBCD. Elife 5 (2016).

42. Taylor A, Smith GR. Unwinding and rewinding of DNA by the RecBC enzyme. Cell 22(2 Pt 2), 447-457 (1980).

43. Ganesan S, Smith GR. Strand-specific binding to duplex DNA ends by the subunits of the Escherichia coli RecBCD enzyme. J. Mol. Biol. 229(1), 67-78 (1993).

44. Taylor AF, Smith GR. RecBCD enzyme is a DNA helicase with fast and slow motors of opposite polarity. Nature 423(6942), 889-893 (2003).

45. Braedt G, Smith GR. Strand specificity of DNA unwinding by RecBCD enzyme. Proc Natl Acad Sci U S A 86(3), 871-875 (1989).

46. Smith GR. How RecBCD enzyme and Chi promote DNA break repair and recombination: a molecular biologist's view. Microbiol. Mol. Biol. Rev. 76(2), 217-228 (2012).

47. Taylor AF, Amundsen SK, Smith GR. Unexpected DNA context-dependence identifies a new determinant of Chi recombination hotspots. Nucleic Acids Res. 44(17), 8216-8228 (2016).

48. Amundsen SK, Sharp JW, Smith GR. RecBCD Enzyme "Chi Recognition" Mutants Recognize Chi Recombination Hotspots in the Right DNA Context. Genetics 204(1), 139-+ (2016).

49. Handa N, Yang L, Dillingham MS, Kobayashi I, Wigley DB, Kowalczykowski SC. Molecular determinants responsible for recognition of the single-stranded DNA regulatory sequence, chi, by RecBCD enzyme. P Natl Acad Sci USA 109(23), 8901-8906 (2012).

50. Amundsen SK, Smith GR. The RecB helicase-nuclease tether mediates Chi hotspot control of RecBCD enzyme. Nucleic Acids Res. 47(1), 197-209 (2019).

51. Taylor AF, Amundsen SK, Guttman M et al. Control of RecBCD enzyme activity by DNA bindingand Chi hotspot-dependent conformational changes. J. Mol. Biol. 426(21), 3479-3499 (2014).

52. Taylor AF, Schultz DW, Ponticelli AS, Smith GR. RecBC enzyme nicking at Chi sites during DNA unwinding: location and orientation-dependence of the cutting. Cell 41(1), 153-163 (1985).

53. Anderson DG, Kowalczykowski SC. The translocating RecBCD enzyme stimulates recombination by directing RecA protein onto ssDNA in a chi-regulated manner. Cell 90(1), 7786 (1997).

54. Spies M, Kowalczykowski SC. The RecA binding locus of RecBCD is a general domain for recruitment of DNA strand exchange proteins. Mol. Cell 21(4), 573-580 (2006).

55. Krajewski WW, Fu X, Wilkinson M, Cronin NB, Dillingham MS, Wigley DB. Structural basis for translocation by AddAB helicase-nuclease and its arrest at chi sites. Nature 508(7496), 416-419 (2014). * Reports the structures of AddAB in complex with different chi-containing DNA substrates, providing insights into the mechanisms for DNA translocation, unwinding and arrest at chi sites.

56. Saikrishnan K, Yeeles JT, Gilhooly NS, Krajewski WW, Dillingham MS, Wigley DB. Insights into Chi recognition from the structure of an AddAB-type helicase-nuclease complex. EMBO J. 31(6), 1568-1578 (2012).

57. Yeeles JT, Gwynn EJ, Webb MR, Dillingham MS. The AddAB helicase-nuclease catalyses rapid and processive DNA unwinding using a single Superfamily $1 \mathrm{~A}$ motor domain. Nucleic Acids Res. 39(6), 2271-2285 (2011).

58. Wilkinson $M$, Troman L, Wan Nur Ismah WA et al. Structural basis for the inhibition of RecBCD by Gam and its synergistic antibacterial effect with quinolones. Elife 5 (2016). 
59. Dziegielewska B, Beerman TA, Bianco PR. Inhibition of RecBCD enzyme by antineoplastic DNA alkylating agents. J. Mol. Biol. 361(5), 898-919 (2006).

60. Sun DY, Hurley LH. Structure-Activity-Relationships of (+)-Cc-1065 Analogs in the Inhibition of Helicase-Catalyzed Unwinding of Duplex DNA. J. Med. Chem. 35(10), 1773-1782 (1992).

61. Villani G, Cazaux C, Pillaire MJ, Boehmer P. Effects of a single intrastrand $d(G p G)$ platinum adduct on the strand separating activity of the Escherichia coli proteins RecB and RecA. FEBS Lett. 333(1-2), 89-95 (1993).

62. Karu $A E$, Linn S. Uncoupling of the recBC ATPase from DNase by DNA crosslinked with psoralen. Proc Natl Acad Sci U S A 69(10), 2855-2859 (1972).

63. Amundsen SK, Spicer T, Karabulut AC et al. Small-molecule inhibitors of bacterial AddAB and RecBCD helicase-nuclease DNA repair enzymes. Acs Chem Biol 7(5), 879-891 (2012). ** Reports the identification and validation of five series of $A d d A B$ and RecBCD small-molecule inhibitors.

64. Aldred KJ, Kerns RJ, Osheroff N. Mechanism of quinolone action and resistance. Biochemistry 53(10), 1565-1574 (2014).

65. Bell JC, Kowalczykowski SC. RecA: Regulation and Mechanism of a Molecular Search Engine. Trends Biochem Sci 41(6), 491-507 (2016).

66. Lin Z, Kong $\mathrm{H}, \mathrm{Nei} \mathrm{M}, \mathrm{Ma} \mathrm{H}$. Origins and evolution of the recA/RAD51 gene family: evidence for ancient gene duplication and endosymbiotic gene transfer. Proc Natl Acad Sci U S A 103(27), 10328-10333 (2006).

67. Horii T, Ogawa T, Nakatani T, Hase T, Matsubara H, Ogawa H. Regulation of SOS functions: purification of E. coli LexA protein and determination of its specific site cleaved by the RecA protein. Cell 27(3 Pt 2), 515-522 (1981).

68. Little JW, Edmiston SH, Pacelli LZ, Mount DW. Cleavage of the Escherichia coli lexA protein by the recA protease. Proc Natl Acad Sci U S A 77(6), 3225-3229 (1980).

69. Jiang $Q$, Karata K, Woodgate R, Cox MM, Goodman MF. The active form of DNA polymerase $V$ is UmuD'(2)C-RecA-ATP. Nature 460(7253), 359-363 (2009).

70. Ghodke $\mathrm{H}$, Paudel BP, Lewis JS et al. Spatial and temporal organization of RecA in the Escherichia coli DNA-damage response. Elife 8 (2019).

71. Chen Z, Yang H, Pavletich NP. Mechanism of homologous recombination from the RecAssDNA/dsDNA structures. Nature 453(7194), 489-484 (2008). * Reports the structure of RecAssDNA and RecA-dsDNA complexes, providing novel insights into the molecular mechanism of RecA multimerisation and function in RecA* filaments.

72. Maslowska KH, Makiela-Dzbenska K, Fijalkowska IJ, Schaaper RM. Suppression of the E. coli SOS response by dNTP pool changes. Nucleic Acids Res. 43(8), 4109-4120 (2015).

73. Gomez-Gomez JM, Manfredi C, Alonso JC, Blazquez J. A novel role for RecA under non-stress: promotion of swarming motility in Escherichia coli K-12. BMC Biol. 514 (2007).

74. Cox MM. Regulation of bacterial RecA protein function. Crit. Rev. Biochem. Mol. Biol. 42(1), 41-63 (2007).

75. Drees JC, Chitteni-Pattu S, Mccaslin DR, Inman RB, Cox MM. Inhibition of RecA protein function by the RdgC protein from Escherichia coli. J. Biol. Chem. 281(8), 4708-4717 (2006).

76. Lee AM, Ross CT, Zeng BB, Singleton SF. A molecular target for suppression of the evolution of antibiotic resistance: inhibition of the Escherichia coli RecA protein by N(6)-(1-naphthyl)ADP. J. Med. Chem. 48(17), 5408-5411 (2005).

77. Wigle TJ, Lee AM, Singleton SF. Conformationally selective binding of nucleotide analogues to Escherichia coli RecA: a ligand-based analysis of the RecA ATP binding site. Biochemistry 45(14), 4502-4513 (2006).

78. Lee AM, Singleton SF. Inhibition of the Escherichia coli RecA protein: zinc(II), copper(II) and mercury(II) trap RecA as inactive aggregates. J. Inorg. Biochem. 98(11), 1981-1986 (2004).

79. Bunnell BE, Escobar JF, Bair KL, Sutton MD, Crane JK. Zinc blocks SOS-induced antibiotic resistance via inhibition of RecA in Escherichia coli. PLoS One 12(5), e0178303 (2017). 
80. Sengupta $S$, Bandyopadhyay S. De novo design of potential RecA inhibitors using multi objective optimization. IEEE/ACM Trans Comput Biol Bioinform 9(4), 1139-1154 (2012).

81. Tiwari V, Tiwari M, Biswas D. Rationale and design of an inhibitor of RecA protein as an inhibitor of Acinetobacter baumannii. J Antibiot (Tokyo) 71(5), 522-534 (2018).

82. Lee AM, Wigle TJ, Singleton SF. A complementary pair of rapid molecular screening assays for RecA activities. Anal. Biochem. 367(2), 247-258 (2007).

83. Wigle TJ, Singleton SF. Directed molecular screening for RecA ATPase inhibitors. Bioorg Med Chem Lett 17(12), 3249-3253 (2007).

84. Wigle TJ, Sexton JZ, Gromova AV et al. Inhibitors of RecA activity discovered by highthroughput screening: cell-permeable small molecules attenuate the SOS response in Escherichia coli. J. Biomol. Screen. 14(9), 1092-1101 (2009).

85. Baell J, Walters MA. Chemistry: Chemical con artists foil drug discovery. Nature 513(7519), 481-483 (2014).

86. Peng Q, Zhou S, Yao F et al. Baicalein suppresses the SOS response system of Staphylococcus aureus induced by ciprofloxacin. Cell. Physiol. Biochem. 28(5), 1045-1050 (2011).

87. Peterson EJ, Janzen WP, Kireev D, Singleton SF. High-throughput screening for RecA inhibitors using a transcreener adenosine 5'-O-diphosphate assay. Assay Drug Dev. Technol. 10(3), 260268 (2012).

88. Aldrich C, Bertozzi C, Georg Gl et al. The Ecstasy and Agony of Assay Interference Compounds. Acs Chem Biol 12(3), 575-578 (2017).

89. Baell JB, Nissink JWM. Seven Year Itch: Pan-Assay Interference Compounds (PAINS) in 2017Utility and Limitations. Acs Chem Biol 13(1), 36-44 (2018).

90. Sexton JZ, Wigle TJ, He Q et al. Novel Inhibitors of E. coli RecA ATPase Activity. Curr Chem Genomics 4 34-42 (2010).

91. Shoichet BK. Interpreting steep dose-response curves in early inhibitor discovery. J. Med. Chem. 49(25), 7274-7277 (2006).

92. Cline DJ, Holt SL, Singleton SF. Inhibition of Escherichia coli RecA by rationally redesigned Nterminal helix. Org Biomol Chem 5(10), 1525-1528 (2007).

93. Yakimov A, Pobegalov G, Bakhlanova I, Khodorkovskii M, Petukhov M, Baitin D. Blocking the RecA activity and SOS-response in bacteria with a short alpha-helical peptide. Nucleic Acids Res. 45(16), 9788-9796 (2017).

94. Azzarito V, Long K, Murphy NS, Wilson AJ. Inhibition of alpha-helix-mediated protein-protein interactions using designed molecules. Nat Chem 5(3), 161-173 (2013).

95. Kalafatovic D, Giralt E. Cell-Penetrating Peptides: Design Strategies beyond Primary Structure and Amphipathicity. Molecules 22(11), (2017).

96. Roy S, Ghosh P, Ahmed I, Chakraborty M, Naiya G, Ghosh B. Constrained alpha-Helical Peptides as Inhibitors of Protein-Protein and Protein-DNA Interactions. Biomedicines 6(4), (2018).

97. Butala M, Zgur-Bertok D, Busby SJ. The bacterial LexA transcriptional repressor. Cell. Mol. Life Sci. 66(1), 82-93 (2009).

98. Zhang AP, Pigli YZ, Rice PA. Structure of the LexA-DNA complex and implications for SOS box measurement. Nature 466(7308), 883-886 (2010). * Reports the strucutre of LexA dimer in complex with SOS box DNA sequence, providing insights into the molecular mechanism of LexA function.

99. Luo Y, Pfuetzner RA, Mosimann S et al. Crystal structure of LexA: a conformational switch for regulation of self-cleavage. Cell 106(5), 585-594 (2001).

100. Neher SB, Flynn JM, Sauer RT, Baker TA. Latent ClpX-recognition signals ensure LexA destruction after DNA damage. Genes Dev. 17(9), 1084-1089 (2003).

101. Bellio P, Mancini A, Di Pietro $L$ et al. Inhibition of the transcriptional repressor LexA: Withstanding drug resistance by inhibiting the bacterial mechanisms of adaptation to antimicrobials. Life Sci. 241117116 (2020). 
102. Roland KL, Little JW. Reaction of LexA repressor with diisopropyl fluorophosphate. A test of the serine protease model. J. Biol. Chem. 265(22), 12828-12835 (1990). 